\title{
The CABANA Debate - Once a Treatment Becomes Popular, it is Hard to Interpret the Results in Medicine
}

\author{
Ozcan Ozeke, Serkan Cay, Firat Ozcan, Serkan Topaloglu, Dursun Aras \\ Department of Cardiology, Health Sciences University, Ankara City Hospital, Ankara, Turkey
}

DOI: https://doi.org/10.17925/EJAE.2019.5.1.17

\begin{abstract}
A trial fibrillation (AF) is the most common clinical arrhythmia and is associated with increased morbidity and mortality. New approaches to AF management, including the use of novel technologies and risk-factor modifications, have the potential to result in better treatment selection and stratified therapy. However, AF prevention has not been emphasised enough in clinical practice. Moreover, we know from experience how hard it is to limit the use of a treatment once it becomes ingrained. The CABANA (Catheter Ablation versus Anti-arrhythmic Drug Therapy for Atrial Fibrillation) trial was a long-awaited, and perhaps delayed, randomised controlled trial (RCT) in AF ablation. The results of CABANA were interpreted differently by cardiologists, neurologists, electrophysiologists and even medical writers. Some electrophysiologists saw the CABANA results as negative, preferring the intention-to-treat (ITT) analysis, whereas others saw it as positive, preferring non-ITT analyses due to high crossover rates. Some cardiologists called the study neutral and said that it was not a game-changer, since they felt that AF ablation only provided symptomatic improvement in daily practice, whereas some authors criticised the electrophysiology community for their emotional approach. The reporting of the CABANA trial by the media was also problematic due to the sensationalism of the reporting. 'Justice delayed is justice denied' is a legal cliché, meaning that if timely justice is not provided to the sufferer, it loses its importance and violates human rights. In medicine, this can translate to 'the RCT delayed is sometimes justice denied'. This article is a collection of comments which are part of the \#CardioTwitter movement, accompanied by some memorable quotes and anecdotes.
\end{abstract}

\section{Keywords}

CABANA trial, atrial fibrillation, catheter ablation, social media, Twitter, \#CardioTwitter, \#CABANA

Disclosures: Ozcan Ozeke, Serkan Cay, Firat Ozcan, Serkan Topaloglu and Dursun Aras have nothing to disclose in relation to this article.

Review Process: Double-blind peer review Compliance with Ethics: This article is an opinion piece and does not report on new clinical data, or any studies with human or animal subjects performed by any of the authors.

Authorship: The named authors meet the International Committee of Medical Journal Editors (ICMJE) criteria

for authorship of this manuscript, take responsibility for the integrity of the work as a whole, and have given final approval for the version to be published.

Received: 27 February 2019

Accepted: 1 April 2019

Citation: European Journal of Arrhythmia

\& Electrophysiology. 2019;5(1):17-22

Corresponding Author: Ozcan Ozeke, Ankara

Sehir Hastanesi, Kardiyoloji Klinigi, Ankara,

06800, Turkey. E: ozcanozeke@gmail.com

Support: No funding was received for

the publication of this article.
Atrial fibrillation (AF) is the most common sustained cardiac arrhythmia in clinical practice, and catheter ablation of AF is currently one of the most commonly performed electrophysiology procedures. Growing experience with ablation techniques, and better AF suppression compared with antiarrhythmic medication, has paved the way for its extended use and indication. The CABANA (Catheter Ablation Versus Anti-arrhythmic Drug Therapy for Atrial Fibrillation) trial was a long-awaited, and perhaps delayed, randomised controlled trial (RCT), ${ }^{1}$ the results of which were presented at the annual scientific meeting of the Heart Rhythm Society 2018 in Boston and published the results in JAMA in March 2019. ${ }^{2}$ The aim of the trial was to test trial whether primary catheter ablation was superior to conventional drug therapy. It included 2,204 patients treated in 10 countries for new onset or undertreated paroxysmal, persistent or long-standing persistent AF who 'warrant therapy' (symptoms or inadequately controlled rates). ${ }^{2}$ Asymptomatic patients comprised only $10 \%$ of the study population. Participants were randomised to catheter ablation, primarily focused on pulmonary vein isolation (PVI), or to drug therapy alone with rate or rhythm control medications. At a median follow-up of 4 years, the difference in the primary endpoint, including death, disabling stroke, serious bleeding or cardiac arrest, between patients assigned ablation and those assigned drug therapy was not significant in the intention-to-treat analysis (ITT) (8.0\% versus 9.2\%; hazard ratio [HR] 0.86; 95\% confidence interval [Cl] 0.65-1.15; $\mathrm{p}=0.30$ ). Despite the neutral finding of the main ITT analysis, a per-protocol (PP) analysis (HR 0.73; 95\% Cl 0.54-0.99) and an as-treated (AT) analysis (7.0\% versus 10.9\%; HR 0.67; 95\% Cl 0.5-0.89) favoured ablation over drug therapy for the primary composite outcome. ${ }^{2}$

A similar phenomenon was observed in the CABANA quality-of-life study. ${ }^{3}$ Contrary to what has previously been seen, whereby the treatment benefits of catheter ablation appear to hit a ceiling at 12 months and decline slowly thereafter, ${ }^{2}$ late attenuation of ablation was not reported in this analysis. ${ }^{3}$ As expected, ablation was associated with a significant reduction in time to first AF recurrence (HR 0.53; 95\% $\mathrm{Cl}$ 0.46-0.61). Patients with the worst quality-of-life assessments at baseline reported the greatest improvement after catheter ablation ( $p$-value for interaction $=0.02$ ). ${ }^{3}$ There were some signals from the CABANA subgroup analysis that patients over 75 years may not benefit as much in terms of mortality, but patients with heart failure seemed to especially benefit. ${ }^{2}$

\section{The CABANA debate}

The results of CABANA trial were interpreted differently by cardiologists, neurologists, electrophysiologists, and even medical writers. ${ }^{4-7}$ While some electrophysiologists see the 
CABANA results as negative, preferring the ITT analysis, ${ }^{8}$ others see them as positive, ${ }^{9}$ preferring the non-ITT analyses due to high crossover rates (see Quote 1-10). ${ }^{10-22}$ Some cardiologists called the study neutral, ${ }^{23}$ and said that it was not a game-changer, since they felt that AF ablation only provided symptomatic improvement in daily practice. Some stressed that the CABANA trial, in its initial presentation, was an observational study that did not actually compare ablation versus drugs as was intended but rather it compared the strategy of ablation versus initial medical therapy with ablation for recurring symptoms (see Quote 4). ${ }^{15,19,24,25}$

The most discussed issue in the CABANA trial was the high numbers of patients who did not receive the treatment they were assigned (see Quote 11). ${ }^{26,27}$ During the conduct of RCTs, it is not uncommon to have protocol violations or inability to assess outcomes. However, the placebo-controlled, double-blind concept that has undoubtedly become the 'gold standard' for studying the efficacy and safety of medical treatments, it is not necessarily free from bias. ${ }^{28,29}$ In an ITT analysis, sometimes called 'analyse as randomised' analysis, participants are analysed on the basis of the treatment arm to which they were initially assigned, regardless of their ultimate treatment exposure, to avoid potential bias due to exclusion of patients. However, when patients do not follow the protocol for their assigned treatment and there are many crossovers, the resultant treatment contamination can produce misleading findings. In such situations, it makes the ITT analysis difficult to interpret and investigators typically conduct non-ITT analyses such as PP and AT analyses. The aim of PP analysis is to identify a treatment effect which would occur under optimal conditions; i.e., to answer the question 'what is the effect if patients are fully compliant?'. This approach may give insight into the efficacy of treatment under optimal conditions; however, its results do not represent the real-life situation and it is likely to show an exaggerated treatment effect. There are also some minor differences between the PP and AT analyses. The PP analysis refers to inclusion of only those patients who strictly adhered to the protocol (using only data from 'perfect' subjects after exclusion of non-compliant patients) and completed the treatment originally planned. Therefore, the exclusion of non-adherers under the PP approach distinguishes it from the AT method, which analyses patients according to the treatment they actually received. ${ }^{30,31}$ However, both of the non-ITT approaches might lose the balance of randomisation and thus are more akin to an observational study. ${ }^{32,33}$

Some authors criticised the use of an antiarrhythmic-drug arm in the CABANA trial as a control group rather than a rate control or no treatment group (see Quote 4), ${ }^{15}$ as the antiarrhythmic-drug arm was very heterogeneous. Since our electrophysiological perception is more valuable than scientific evidence, some criticised the electrophysiology community for their emotional approach (see Quote 10). ${ }^{21,22,25}$ Even if the outcomes of CABANA, in both the ITT and non-ITT analyses, would have been found not superior to control group, would it also change the practical approach in electrophysiology practice? ? $34,35^{2}$

\section{Once a treatment becomes popular, it is hard to interpret the results in medicine}

We know from experience how hard it is to limit the use of a treatment once it becomes ingrained. In 2002, The New England Journal of Medicine published an interesting study, in which many patients reported symptomatic relief after undergoing arthroscopy of the knee for osteoarthritis, but it was unclear how the procedure achieves this result. The study showed that the outcomes after arthroscopic lavage or arthroscopic debridement for osteoarthritis were no better at relieving pain or improving function than those after a placebo/sham procedure. ${ }^{5,36}$ However, many surgeons and medical societies disputed the study and pressed insurance companies to maintain coverage of the procedure. In contrast to the anticipated impact of the COURAGE (Clinical Outcomes Utilizing Revascularization and Aggressive Drug Evaluation) trial on daily cardiology practice, Garg et al. found lower rates of optimal medication use in patients with percutaneous coronary intervention after its publication. In the post-truth age, ${ }^{35}$ the medicine has always existed somewhat uncomfortably in the dual worlds of science and belief. Eminence-based medicine refers to a clinical decision that is made by relying purely on the opinion of a medical specialist or any prominent health professionals rather than relying on critical appraisal of scientific evidence available. ${ }^{37}$ This is probably due to several reasons such as a self-serving bias, the Dunning-Kruger effect, ${ }^{38}$ or other unethical medical professionalism (see Quotes 12-15). ${ }^{39-43}$ There is a tendency to overestimate the value and underestimate the risk of the intervention for many proceduralists and ablationists in medicine..$^{44,45}$ Moreover, in some cases, experts say that doctors are motivated to use stents/ablation procedures/orthopaedic implants for financial reasons, because of the large revenue streams that these procedures can bring hospitals (see Quotes 14 and 15). ${ }^{46,47}$ It is likely that overtreatment and unnecessary surgeries and procedures continue in daily practice..$^{20}$ Moreover, the training programmes today train ablationists and implanters, not electrophysiologists (see Quote 16).39,48

Although nowadays performed on a routine basis, AF ablation is associated with the potential for major complications, even in experienced high-volume centers. ${ }^{49}$ Despite the fact that adverse-event rates were relatively low in both patient groups in the CABANA trial, the success rates and complication rates in the CABANA trial might not reflect that of the real-world rate. Today, the most dreadful and lethal complication of AF ablation is atrioesophageal fistula, which remains a very rare but serious complication despite the incorporation of protective measures and increased technical experiences. A high index of suspicion is recommended in patients who develop constitutional symptoms or sudden onset chest pain or neurological symptoms that start days or weeks after AF ablation. However, few centres measure outcomes or success of ablation and follow patients for future atrioesophageal fistula complication (see Quote 17 and 18);50,51 therefore, it is usually underdiagnosed and likely under-reported due to a number of factors including misdiagnosis. Do the patients know that AF is not deadly heart disease but that ablation might cause deadly complication such as atrioesophageal fistula (see Quotes 19-22) ?18,51-4 Moreover, almost half of patients and physicians believed AF ablation would eliminate the need for anticoagulation while the great majority of both groups believed AF ablation would decrease stroke rates. ${ }^{55}$ We must be sure that the patient's symptoms are related to AF itself and not palpitations, fear or anxiety (see Quotes 23 and 24). ${ }^{48,56,57}$ There is no doubt that AF ablation is superior over antiarrhythmic drugs in terms of arrhythmia recurrence, symptoms and quality of life (RAAFT-2 trial, ${ }^{58}$ CAMTAF trial ${ }^{59}$ ); however, to date, there are no RCTs with hard clinical endpoints such as mortality or stroke. After the CABANA trial, the topic of a sham-controlled trial continued to come up in discussions.

Almost every cardiac electrophysiologist has experience of patients who greatly benefited from catheter ablation of $\mathrm{AF}$, or have even seen an almost curative effect in some patients. On the other hand, overused ablation procedures in unselected patient populations is a reality in daily practice. Additionally, some patients reported relief of symptoms even in cases where the arrhythmia persisted despite the ablation.5,60-2 These cases can be explained by the placebo, the pseudo-placebo or neuromodulation effects of ablation (see Quotes 25 and 26). . $21,35,60,63-8$ 
Because catheter ablation of AF has never been studied in a randomised, blinded fashion, we cannot know whether patients experience fewer symptoms after ablation because subjective symptoms frequently decrease following a procedure, or whether the ablation itself was beneficial. ${ }^{42,64,69,70}$ Most doctors believe that a sham trial would be unethical; ;1-3 however, it might also be unethical to apply the ablation procedure to patients that may really not benefit from an invasive procedure (see Quote 26).68,70 Indeed, neither physicians nor patients would like to enrol in a sham procedure group in a real life; 9,74 nevertheless, physicians should keep in mind, at least conceptually, ${ }^{75}$ the placebo possibility of a new invasive procedure before extensive use of it. ${ }^{18,55}$ Beyond the possible placebo effect, the pseudo-placebo effect might also be relevant in clinical practice..$^{75}$ While a positive effect of ablation may be seen in RCTs performed in highly selected patients with evidence of pulmonary vein triggered AF (not only based on presence of pulmonary vein potentials), ${ }^{76}$ that same positive effect might not found in sham procedures where the RCT is were performed in patients with extensive substrate or with seconder $\mathrm{AF}_{1}{ }^{77}$ as did in daily practice. This debate seems to continue until the personalised patient-specific and mechanism-based AF classification would be common use in daily practice instead of temporal AF classification (see Quote 27). ${ }^{78}$

\section{Conclusion}

For many years, both physicians and patients have had a 'more is better' attitude (see Quote 28). ${ }^{34,79,80}$ In contrast to this, in 2010, Grady and Redberg published an editorial titled 'Less is More: How Less Health Care Can Result in Better Health'. ${ }^{81}$ We must re-enter the age of not nihilist but empathetic medical conservative, as was done in the past. ${ }^{82}$ The extension of clinical indications for invasive procedures with optimistic theory without waiting for the results of a delayed RCT is another hidden problem in modern medicine. Clinical decisions are ideally based on evidence generated from multiple RCTs evaluating clinical outcomes, but historically, few clinical guideline recommendations have been based entirely on this type of evidence. Among recommendations from major cardiovascular society guidelines from 2008-18, the proportion supported by evidence from RCTs remains small. ${ }^{83}$ The real CABANA debate is why such a trial was not done 20 years ago before the extensive acceptance of $\mathrm{AF}$ ablation by electrophysiologists, ablationists and even patients.

'Justice delayed is justice denied' is a legal cliché meaning that if timely justice is not provided to the sufferer, it loses it importance and violates human rights. In medicine, this can translate to 'the RCT delayed is justice denied'. ${ }^{35}$ It is clear that if approval of procedures becomes too stringent, it could quash the development of potential breakthrough therapies. The barriers to change established practice may prevent or impede progress in science and technology (see Quotes 29-32). ${ }^{84-7}$ Therefore, the technological progress in medicine has been a mixed blessing (see Quotes 33 and 34). ${ }^{88,89}$ We need a balance between the two fundamental ethical obligations, a Hippocratic one and a scientific one (see Quotes 35 and 36). ${ }^{90-2}$

Dr Haissaguerre and Dr Chen deserve special thanks and appreciation for their breakthrough innovation in the field of electrophysiology. ${ }^{36,93,94}$ The RCTs of therapeutic strategies, in particular those involving invasive procedures, are among the most challenging to design and execute, therefore, Dr Packer deserves special thanks and appreciation for the CABANA trial (see Quote 37).2,95,96 The CABANA trial provides a wealth of additional data regarding the comparative benefits and risks of catheter ablation versus drug therapy to inform this process. ${ }^{64}$ The initial and critical step toward meaningful outcomes with AF ablation is proper patient selection in both daily practice and RCTs to prevent the placebo and pseudo-placebo effects (see Quote 38 ). ${ }^{75,97}$ While $19.4 \%$ of the patients randomised to catheter ablation underwent repeat procedures in the CABANA trial, it is also important to note that more than $50 \%$ of patients randomised to ablation had a recurrence of AF over 4 years, and some of these patients may require repeat ablations in the future. ${ }^{64}$ It is the responsibility of the electrophysiology community to educate patients and referring physicians regarding the true benefits of AF ablation (see Quotes 38 and 39.97-9 Shared decision making between the cardiologist and the patient is critical in the care of patients with AF in a very patient-centric way. ${ }^{57}$ This will also certainly help to rebuild the trust in a patient-physician relationship (see Quotes 40 and 41). ${ }^{29,35,57,65,74,82,100}$

\section{CardioTweets and \#CardioTwitter with memorable quotes and anecdotes}

Social media are dynamic and interactive computer-mediated communication tools that have high penetration rates in the general population. There has been a recent increase in the percentage of cardiovascular professionals to engage with others and stay current with evidence, learn new techniques and share practice strategies. ${ }^{101}$ It is positively transforming society and medicine by providing the democratisation of medicine. ${ }^{102,103}$ Here we have compiled comments from \#CardioTwitter and added some memorable quotes and anecdotes

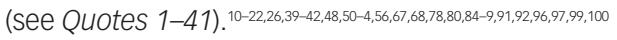

Quote 1: Douglas Packer 'You can't get benefit from a therapy if you don't get the therapy.'10,11

Quote 2: John Day 'The big take away from the CABANA Study is that ablation performed remarkably well. It is especially an attractive option for the patient with AF symptoms that don't respond to medications. Should everyone get an ablation? Of course not. If you can't reverse AF with an optimized lifestyle then it may be a reasonable option. For those in whom drugs don't work or cause side effects, then ablation is definitely a logical choice.' ${ }^{\prime 2,13}$ [sic]

Quote 3: J Brian DeVille 'It is very difficult to tell the full story of a study that has been developing for over 10 years in a tweet, especially in a

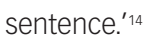

Quote 4: Jose Luis Merino 'The CABANA trial has generated significant controversy about whether it resulted in positive or neutral ablation results. This controversy mostly comes from the high rate of crossovers from the antiarrhythmic drug arm to the ablation arm. In general, comparison between different treatments have to be done in an intention-to-treat basis since this is what finally matters the clinician in order to choose the initial therapy. However, this kind of analysis works fairly well in double-blinded studies but maybe it not as good in unblinded ones, in particular when therapies being compared are very different. In this latter case, it may be difficult to keep patients on a treatment which works worse than the other. Following crossover, the trial may end with many patients (in particular with those who are doing poorly with the initially allocated treatment) benefiting for the alternative treatment while this benefit is being attributed to the initial one. In addition, what is questioned in the CABANA trial is not the reduction in symptoms or AF recurrences with ablation but hard outcomes such as hospitalization for heart failure, stroke and death. This is relevant since in the absence of proven prognostic benefits most patients should receive a first attempt with the simplest treatment to control their symptoms. Finally, most antiarrhythmic drugs have provided no prognostic benefit in AF patients and even a trend towards a worse outcome was suggested in some studies such as the AFFIRM trial. All these factors justify conducting a new trial comparing ablation versus no antiarrhythmic treatment in patients 
with no or atrial fibrillation symptoms which do not warrant treatment. In this latter population, both evaluation of 'pure' prognostic benefits of ablation and a low risk of treatment crossover are warranted and the trial would answer the one-million-dollar question if ablation should be offered in first place to most patients regardless of their symptoms. ${ }^{15}$

\section{Quote 5: Milton Packer 'So if I personally had symptomatically} troubling AF, I would probably decide to undergo catheter ablation if initial drug therapy was unsatisfactory. However, the point of this post is not whether patients should or should not undergo ablation. Its entire point is to ask whether it is proper for electrophysiologists to distort and deny the facts to support a preconceived notion. Why not be honest? They could simply say that patients are likely to prefer ablation, even though there is no evidence that ablation prevents any of the serious consequences of atrial fibrillation. Why would you design a randomized trial if you are going to analyze it in a way that is inconsistent with randomization? We do not perform or accept the PP analyses in trials of drug therapy. ${ }^{\prime 16}[\mathrm{sic}]$

Quote 6: Edip Gurel 'I think there are patients who benefit from catheter ablation. The problem is to define this population and select the ones who would benefit the most. Not an easy task. CABANA may not be a victory but it is a step forward. ${ }^{17}$ [sic]

Quote 7: Edward J Schloss 'Critical messaging of trials like ORBITA and CABANA should not be 'treatment (PCI/AFAbl) doesn't work', but rather, "how do we select appropriate patients for these treatments?"'18

Quote 8: Atul Verma 'CABANA is the gift that keeps giving... ITT for the doubters and PP for the believers. AFFIRM did not kill the pursuit of sinus and neither will CABANA. The science continues... ${ }^{19}[\mathrm{sic}]$

Quote 9: William Sauer 'The trial results appear to confuse many patients and physicians. AF patients should be referred to electrophysiologist [sic] who can explain the details at the initial consult and then decide for ablation themselves with a shared decision-making tool. ${ }^{20}$

Quote 10: John Mandrola 'A core reason trials produce a higher level of evidence than observational studies is randomization. This is why readers of CABANA must acknowledge that any findings other than the ITT analysis are observational. ITT preserves randomization. Even though the AT comparison makes sense, one must stick to the rules of science. Why are the results of trials emotional? Why not celebrate the knowledge gained? Why is it disappointing that ablation did not reduce outcomes?'21,22 [sic]

Quote 11: Bogdan Enache 'I seriously don't understand the 'problem with crossover' in \#CABANA; [...] Authors planned and powered for a crossover rate of either $25 \%$ (according to the main manuscript) or 25-30\% (according to the published protocol) [...] 27\% of control arm got AF ablation. Why is this dramatic? ${ }^{26}$

Quote 12: Abraham Maslow 'If the only tool you have is a hammer, to treat everything as if it were a nail.. ${ }^{39}[\mathrm{sic}]$

Quote 13: Fulton Sheen 'If you do not live what you believe, you will end up believing what you live.'40

Quote 14: Upton Sinclair 'It is difficult to get a man to understand something when his salary depends on his not understanding it.' ${ }^{\prime 41}$
Quote 15: Sanjay Kaul 'In the battle between revenue-based medicine vs evidence-based medicine, remuneration always wins. ${ }^{42}$ [sic]

Quote 16: Mark E Josephson 'Training programs today train 'ablationists' and 'defibrillationists' (a.k.a. implanters), not electrophysiologists. There has become a total dependence on technology, but no understanding of the limitations of that technology. Dependence on technology has resulted in inappropriate and/or excessive radiofrequency lesions because of the lack of understanding of both the electrophysiology and the limitations of the technology being used. I believe that training for AF ablation in many laboratories involves no mechanistic or electrophysiologic approach but mainly involves designing ways to complete and connect dots around and between the pulmonary veins. ${ }^{\prime}{ }^{48}$

Quote 17: Anonymous 'If you don't look for it, you probably won't see it.' ${ }^{50}$

Quote 18: Bayazid Bastami 'The thing we tell of can never be found by seeking, yet only seekers find it. ${ }^{51}[\mathrm{sic}]$

Quote 19: Anonymous 'You know which operation has the highest complication rate? The one that's not indicated. ${ }^{52}$

Quote 20: Joseph Stalin 'One death is a tragedy; one million is a statistic. $^{.53}$

Quote 21: Ibn Sina (Avicenna) 'Width of life is more important than length of life. ${ }^{54}$

Quote 22: Douglas Packer 'Physicians have a different point of view about endpoints than patients: MDS - mortality vs patients - QOL.' ${ }^{18}$ [sic]

Quote 23: Mark E Josephson 'Our programs no longer teach our electrophysiology fellows how to be 'physicians' who can listen to their patients' complaints and formulate a plan of action that is based on scientific evidence (both pro and con) and sound judgment. '.48

Quote 24: Richard Baron 'One of the most important questions any patient should ask anytime they're thinking about having a surgical procedure is, what happens if I don't do this? Are there other options? What are the risks?'56

Quote 25: Blaise Pascal 'The heart has its reasons which reason does not understand. ${ }^{67}[\mathrm{sic}]$

Quote 26: Rita Redberg 'People say, "It's unethical to do a sham-controlled trial." I think it's unethical not to!'68

Quote 27: Ibn Sina (Avicenna) 'Absence of understanding does not warrant absence of existence. ${ }^{78}[\mathrm{sic}]$

Quote 28: Lewis Thomas 'The dilemma of modern medicine, and the underlying central flaw in medical education and, most of all, in the training of interns, is the irresistible drive to do something, anything. It is expected by patients and too often agreed to by their doctors, in the face of ignorance. ${ }^{180}$

Quote 29: Richard Hooker 'Change is not made without inconvenience, even from worse to better. ${ }^{\prime} 84$ 
Quote 30: Albert Einstein 'A person who never made a mistake never tried anything new.'85

Quote 31: André Gide 'One doesn't discover new lands without consenting to lose sight, for a very long time, of the shore. ${ }^{.86}$ [sic]

Quote 32: Fatih Sultan Mehmet 'In order to see the boundaries of the probabilities, need to try impossible.' ${ }^{\prime 8}[\mathrm{sic}]$

Quote 33: Arthur William Galston 'Nothing that you do in science is guaranteed to result in benefits for mankind. Any discovery, I believe, is morally neutral and it can be turned either to constructive ends or destructive ends. That's not the fault of science. ${ }^{\prime 8}$

Quote 34: Larry Husten '300+ years later and nothing has changed. Do we control technology, or does technology control us? ${ }^{\prime 89}$

Quote 35: Hippocratic Oath 'First do not harm. ${ }^{\prime 91}$ [sic]

Quote 36: Suleiman the Magnificent 'The people think of wealth and power as the greatest fate, But in this world a spell of health is the best state. ${ }^{\prime 92}[\mathrm{sic}]$

Quote 37: Stuart Mary 'This is a landmark study with the heft of the Framingham Study. Insights from this will be coming out, forever.'96
Quote 38: Christiaan Barnard 'I have saved the lives of 150 people from heart transplantations. If I had focused on preventive medicine earlier, I would have saved 150 million. ${ }^{97}$

Quote 39: William Osler 'The good physician treats the disease; the great physician treats the patient who has the disease.' ${ }^{\prime 9}$

Quote 40: Alice K Jacobs (Presidential opening speech at AHA 2004) 'Each year we gather at these Sessions, the world's largest meeting of scientists and healthcare professionals dedicated to the basic, clinical, and population science of cardiovascular disease and stroke. To be sure, we will learn about the emerging science and clinical practice of cardiovascular disease over the next four days. But there is an internal disease of the heart that confronts us as scientists, as physicians, and as healthcare professionals. It is a threat to us all-insidious and pervasive - and one that we unknowingly may spread. This threat is one of the most critical issues facing our profession today. How we address this problem will shape the future of medical care. This issue is the erosion of trust. At this time, when more and more public and private institutions have fallen in public esteem, restoring trust in the healthcare professions will require that we understand the importance of trust and the implications of its absence.' 100

Quote 41: Julie Gerberding 'You can manage people if they trust you. ${ }^{\prime 100}$
1. Packer DL, Mark DB, Robb RA, et al. Catheter ablation versus antiarrhythmic drug therapy for atrial fibrillation (CABANA) trial: antiarrhythmic drug therapy for atrial fibrillation (CABAN
study rationale and design. Am Heart J. 2018;199:192-9.

2. Packer DL, Mark DB, Robb RA, et al. Effect of catheter ablation vs antiarrhythmic drug therapy on mortality, stroke, bleeding and cardiac arrest among patients with atrial fibrillation: The CABANA randomized clinical trial. JAMA. 2019;321:1261-74.

3. Mark DB, Anstrom KJ, Sheng S, et al. Effect of Catheter ablation vs medical therapy on quality of life among patients with atrial fibrillation: The CABANA randomized clinical trial. JAMA 2019;321:1275-85.

4. Vergara G. \#CABANA more questions than answers. Not sure it answered what it was supposed to answer. very very muddy results. 2018. Available at: https://twitter.com/drgastonvergara/ results. 2018. Available at: https://twitter.com/drgaston).
status/994960253925150721 (accessed 11 June 2019).

5. Gupta A. Five reputable sites. $\$ 4.5$ billion business. Yet two completely opposite interpretations of today's \#CABANA completely opposite interpretations of today's \#CABANA
(atrial fibrillation ablation vs med) trial results. Can someone (atrial fibrillation ablation vs med) trial results. Can someone make sense of this? 2018. Avallable at. https.//twitter.com/ AtulGupta_MD/status/994710193878589442 (accessed 11 June 2019)

6. Santangeli P. Key methodological point - when crossover rates are that high only on treatment analyses have practica value. ITT is a nice exercise to do for statistical purists but don't help doctors taking care of patients. 2018. Available at: https://twitter.com/Dr_Santangeli/status/994598112961458176 (accessed 11 June 2019).

7. Enache B. My take: \#CABANA does not change a single person's opinion on AF ablation. \#EHRA2019. 2019. Available at: https://twitter.com/bogdienache/status/1107220797368733696 (accessed 11 June 2019)

8. Packer M. The CABANA Trial: an honourable view. Eur Heart s. 2018:39:2770.

9. Providencia R, Adragao P. Science deserves justice: The results of the CABANA trial are positive and support catheter ablation of atrial fibrillation for reducing mortality and hospitalizations. Rev Port Cardiol. 2019;38:245-50.

10. Packer DL. CABANA: Atrial Arrhythmias in Catheter Ablation vs. Antiarrhythmic Drug For AFib. Presented at: Heart Rhythm Society Scientific Session, Boston, MA, USA, May, 2018.

11. Healthy Dialogues. Discussion on CABANA Research Results at HRS2018. 2018. Available at: www.youtube.com/ watch?v=knWwUBeylQ8 (accessed 11 May 2018).

12. Day J. 9 Key Findings of the CABANA Study. 2018. Available at: https://drjohnday.com/cabana-study/ (accessed 11 June 2019).

13. Day J. 9 reasons why ablation beats drugs for afib: CABANA study results. 2018. Available at: https://twitter.com/ study results. 2018. Available at: https://twitter.com/
drjohndayMD/status/995274778918432769 (accessed 11 June 2019).

14. DeVille JB. Coping with CABANA: Look beyond the headlines. 2018. Available at: www.thehearthospitalbaylor.com/Pages/ Coping-with-CABANA.aspx (accessed 11 June 2019).

15. Merino JL. The commentary-opinion about the CABANA trial. Personal communication with Ozeke 0, 11 June 2018.

16. Packer M. Unbelievable! Electrophysiologists Embrace 'Alternative Facts'. 2018. Available at: www.medpagetoday.com/ blogs/revolutionandrevelation/72905 (accessed 11 June 2019).

17. Gurol E. I think there are patients who benefit from catheter ablation. The problem is to define this population and select the ones who would benefit the most. Not an easy task. \#EPeeps keep your heads up. CABANA may not be a victory but it is a step forward. 2018. Available at: https://twitter.com/guroledip/ status/994770968320016384 (accessed 11 June 2019).

8. Schloss EJ. Critical messaging of trials like \#ORBITA and \#CABANA should not be - Treatment (PCI/AFAbl) doesn't work. But rather, - How do we select appropriate patients for these treatments? 2018. Available at: https://twitter.com/EJSMD/ status/994596144155189250 (accessed 10 May 2019).

19. Verma A. \#CABANA is the gift that keeps giving....IT for the doubters and Per protocol for the believers. AFFIRM did not kill the pursuit of sinus and neither will CABANA. The science continues... \#HRS2018. 2018. Available at: https://twittercom/ atulverma_md/status/994573806856372224 (accessed 11 June 2019).

20. Sauer W. Maybe the best message from \#CABANA: The tria results appear to confuse many patients and physicians. AF patients should be referred to EPs who can explain the details at the initial consult and then decide for ablation themselves with a shared decision making tool. 2018. Available at: https:// twitter.com/True_EP/status/995340670981177344 (accessed 11 June 2019).

21. Mandrola J. I'm considering putting this thread in my column: namely, why are THE RESULTS of trials emotional? Why not celebrate the knowledge gained? Why is it disappointing that ablation did not reduce outcomes? 2018. Available at: https:// twitter.com/drjohnm/status/995295157430964224 (accessed 11 June 2019).

22. Mandrola J. CABANA: Initial thoughts on AF ablation vs drugs. 2018. Available at: www.medscape.com/ viewarticle/896573\#vp 3 (accessed 11 June 2019).

23. Markides V. CABANA - the (not so) neutral study. Eur Heart J 2018;39:2769

24. Mandrola J. Overdiagnosis and overtreatment. 2016 Available at: www.drjohnm.org/2016/01/overdiagnosis-andovertreatment/ (accessed 11 June 2019)

25. Mandrola J. Musings on the CABANA trial - AF ablation vs drugs. 2018. Available at: www.drjohnm.org/2018/05/musingson-the-cabana-trial-af-ablation-vs-drugs/ (accessed 11 June 2019).

26. Enache B. I seriously don't understand the "problem with crossover" in \#CABANA: they planned and powered for a crossover rate of either $25 \%$ (according to the main manuscript) or $25-30 \%$ (according to the published protocol) $-\sim 27 \%$ of control arm got AF ablation Why is this dramatic? 2018. Available at: https://twitter.com/bogdienache/ 2018. Available at. https.//twitter.com/bogdienache/

27. Devireddy C. Science shouldn't be swayed by personal bias. If a procedure shows no benefit in a proper sham RCT, isn't the greater wrong in exposing pts to invasive risk $\mathrm{W} /$ no true benefit? That's the purpose of sham RCTs. Don't underestimate an informed patient's ability to choose. 2018. Available at: https://twitter.com/drdevireddy/status/995512552879738880 (accessed 11 June 2019).

28. Ranganathan P, Pramesh CS, Aggarwal R. Common pitfalls in statistical analysis: Intention-to-treat versus per-protocol analysis. Perspect Clin Res. 2016;7:144-6.

29. Sussman JB, Hayward RA. An IV for the RCT: using instrumenta variables to adjust for treatment contamination in randomised controlled trials. BMJ 2010:340:c2073

30. Altman DG. Better reporting of randomised controlled trials: the CONSORT statement. BMJ. 1996;313:570-1.

31. Sainani KL. Making sense of intention-to-treat. PMR. 2010;2:209-13

32. Pocock SJ, Collier TJ. Statistical appraisal of 6 recent clinica trials in cardiology: JACC State-of-the-Art Review. JACC. 2019;73:2740-55.

33. Noseworthy PA, Gersh BJ, Kent DM, et al. Atrial fibrillation ablation in practice: assessing CABANA generalizability. Eur Heart J. 2019;40:1257-64.

34. Alasnag M. \#CABANA anticipated results Negative for primary endpoint of MACE Crossover rate high Recurrence rate High Disappointment ...And I bet ablation rate will NOT change this year. 2018. Available at: https://twitter.com/mirvatalasnag/ year. 2018. Available at: https://twitter.com/mirvatalas
status/994586243894841344 (accessed 11 June 2019)

35. Ozeke O, Cay S, Ozcan F, et al. Post-truth era and cardiology: After ORBITA, before CABANA. Indian Heart J. 2018;70:439-42.

36. Moseley JB, O'Malley K, Petersen NJ, et al. A controlled trial of arthroscopic surgery for osteoarthritis of the knee. N Eng/ $J$ Med. 2002;347:81-8

37. Isaacs $D$, Fitzgerald $D$. Seven alternatives to evidence based medicine. BMJ. 1999;319:1618

38. Simons DJ. Unskilled and optimistic: overconfident predictions despite calibrated knowledge of relative skill. Psychon Bull Rev. 2013;20:601-7.

39. Wiktionary. if all you have is a hammer, everything looks like a nail. Available at: https://en.wiktionary.org/wiki/if_all_you_ have_is_a_hammer,_everything_looks_like_a_nail (accessed 11 June 2019)

40. Sheen FJ. If you do not live what you believe, you will end up believing what you live. Available at: www.azquotes.com/ quote/867502 (accessed 11 June 2019).

41. Sinclair U. It is difficult to get a man to understand something, when his salary depends on his not understanding it. Available at: www.goodreads.com/quotes/21810-it-is-difficult-to-get-aman-to-understand-something (accessed 11 June 2019)

42. Husten L. CABANA: no outcome $s$ benefit in first big trial of AF ablation. http://www.cardiobrief.org/2018/05/10/ cabana-no-outcomes-benefit-in-first-big-trial-of-af-ablation/ ... 2018. Available at: https://twitter.com/cardiobrief/ status/994652019339251712 (accessed 11 June 2019).

43. Abdullah JM. The eight stages of trust and "Amanah" in medicine and the Dunning-Kruger effect. Malays I Med Sci. 2014;21:1-3.

44. Hoffmann TC, Del Mar C. Clinicians' expectations of the benefits and harms of treatments, screening, and tests: a systematic review. JAMA Intern Med 2017:177:407-19.

45. Arbelo E, Brugada J, Hindricks G, et al. The atrial fibrillation ablation pilot study: a European Survey on Methodology and results of catheter ablation for atrial fibrillation conducted by the European Heart Rhythm Association. Eur Heart J. 2014;35:1466-78

46. Liu JJ, Bell CM, Matelski J, et al. Payments by US pharmaceutical and medical device manufacturers to US medical journal editors: retrospective observational study. BMJ. 2017;359:j4619.

47. Campbell EG, Gruen RL, Mountford J, et al. A national survey of physician-industry relationships. N Eng/ I Med. 
2007;356:1742-50.

48. Josephson ME. Electrophysiology at a crossroads: A revisit. Heart Rhythm. 2016;13:2317-22

49. Deshmukh A, Patel NJ, Pant S, et al. In-hospital complications associated with catheter ablation of atrial fibrillation in the United States between 2000 and 2010: analysis of 93801 procedures. Circulation. 2013;128:2104-12.

50. ScienceDaily. If you're not looking for it, you probably won't see it. 2018. Available at: www.sciencedaily.com/ releases/2013/07/130719112134.htm (accessed 11 June 2019).

51. Wikiquote. Bastami B. Available at: https://en.wikiquote.org/ wiki/Bayazid_Bastami (accessed 11 June 2019).

52. Motameni A. "You know which operation has the highest complication rate? The one that's not indicated". 2018. Available at: https://twitter.com/A_Motameni/ status/997314647043772416 (accessed 11 June 2019).

53. Wikiquote. Stalin J. Available at: https://en. wikiquote.org/wiki/ Joseph_Stalin (accessed 11 June 2019).

54. Avicenna. Width of life is more important than length of life. Available at: https://www.azquotes.com/quote/1463241 (accessed 11 June 2019).

55. Badin A, Parr AR, Banga S, et al. Patients' and physicians' perceptions regarding the benefits of atrial fibrillation ablation. Pacing Clin Electrophysiol. 2017;40:362-71.

56. Neighmond P. Popular surgery to ease chronic shoulder pain called into question. 2017. Available at: www.wxxinews.org/ post/popular-surgery-ease-chronic-shoulder-pain-calledquestion (accessed 11 June 2019).

57. Hills MT. The transformative power of understanding and trust in AF care: How doctors can provide better treatment by understanding the hearts - and minds - of AF patients. I Cardiovasc Electrophysiol. 2018;29:641-2.

58. Morillo CA, Verma A, Connolly SJ, et al. Radiofrequency ablation vs antiarrhythmic drugs as first-line treatment of paroxysmal atrial fibrillation (RAAFT-2): a randomized trial. JAMA. 2014;311:692-700.

59. Hunter RJ, Berriman TJ, Diab I, et al. A randomized controlled trial of catheter ablation versus medical treatment of atrial fibrillation in heart failure (the CAMTAF trial). Circ Arrhythm Electrophysiol. 2014;7:31-8.

60. Ozeke O, Cay S, Ozcan F, et al. Similarities between the renal artery and pulmonary vein denervation trials: Do we have to use sham procedures for atrial fibrillation catheter ablation trials? Int J Cardiol. 2016;211:55-7.

61. Björkenheim A, Brandes A, Magnuson A, et al. Assessment of atrial fibrillation-specific symptoms before and 2 years after atrial fibrillation ablation: Do patients and physicians differ in their perception of symptom relief? JACC Clin Electrophysiol. 2017:3:1168-76.

62. Hindricks $\mathrm{G}$, Piorkowski $\mathrm{C}$, Tanner $\mathrm{H}$, et al. Perception of atrial fibrillation before and after radiofrequency catheter ablation: relevance of asymptomatic arrhythmia recurrence. Circulation. relevance of asymptor
2005:112:307-13.

63. Ozeke O, Aras D, Cay S, et al. Perception paradox between the doctors and patients in the industrial-bureaucratic age of medicine: Defensive versus offensive medicine in anticoagulation and atrial fibrillation ablation. Pacing Clin Electrophysiol. 2017;40:979-80.

64. Albert CM, Bhatt DL. Catheter ablation for atrial fibrillation: Lessons learned from CABANA. JAMA. 2019;321:1255-57.

65. Suh W. And 40 years after the first PTCA to show that stenting in a single vessel CAD may not be as good as we all thought. \#ORBITA Will we have to wait 40 years for an AF ablation sham controlled RCT? \#HRS2018 \#CABANA. 2018. Available at: https://twitter.com/willsuh76/status/994934430094389248 (accessed 11 June 2019).

66. Mandrola J. This means we NEED an \#Orbita-AF trial. \#HRS2018. 2018. Available at: https://twitter.com/drjohnm/ status/994573735830151169 (accessed 11 June 2019).

67. Pascal B. The heart has its reasons of which reason knows nothing. Available at: www.brainyquote.com/quotes/blaise pascal 132990 (accessed 11 June 2019).

68. Alderighi C.As Dr Rita Redberg said at the Lown Conference: "People say, 'It's unethical to do a sham-controlled trial.' I think it's unethical not to!" 2018. Available at: https://twitter com/camialderighi/status/997823201475465216 (accessed 11 June 2019).

69. Gibson CM. Dr Deepak Bhatt's summary of \#CABANA on ACC website: trial is only single-blinded (not to intervention received). That may have driven the high crossover rates and can confound assessment of the various endpoints ... should prompt consideration of a sham-controlled trial. 2018. Available at: https://twitter.com/CMichaelGibson/ status/994925647582543873 (accessed 11 June 2019).

70. American College of Cardiology. Catheter ablation vs antiarrhythmic drug therapy in atrial fibrillation - CABANA. 2018. Available at: www.acc.org/latest-in-cardiology/clinical2018. Available at: Www. acc.org/latest-in-cardiology//linica-

71. Natale A. It is too bad that analysts blindly report the words of who they talk to. If you say "ablation", you should be honest and say that it reduces events. Then the ITT analysis is a different issue. 2018. Available at: https://twitter.com/ andreanatalemd/status/995363238593400834 (accessed 11 June 2019).

72. Verma A. I would tend to agree. \#CABANA is a large and complex trial with much to unpack. A sham trial would not be my chosen next step. 2018. Available at: https://twitter.com/ atulverma_md/status/994597389162889216 (accessed 11 June 2019).

73. Al-Ahmad A. Lots to discuss and learn with CABANA. Don't agree with sham trial. 2018. Available at: https://twitter.com/ aalahmadmd/status/994650638788235265 (accessed 11 June 2019).

74. Hills M. Maybe, but I personally could never recommend for a patient to undergo a sham procedure. That is cruel to even consider. Put yourself in the patient's shoes. You would not want that either. 2018. Available at: https://twitter.com/mhills/ status/995366032578613248 (accessed 11 June 2019).

75. Ozeke O, Cay S, Ozcan F, et al. The placebo effect in atria fibrillation ablation: Placebo or pseudo-placebo effect? Pacing Clin Electrophysiol. 2018;41:872-3

76. Kuck KH, Hoffmann BA, Ernst S, et al. Impact of complete versus incomplete circumferential lines around the pulmonary veins during catheter ablation of paroxysmal atrial fibrillation: Results from the Gap-Atrial Fibrillation-German Atrial Fibrillation Competence Network 1 Trial. Circ Arrhythm Electrophysiol. 2016;9:e003337.

77. Marrouche NF, Wilber D, Hindricks G, et al. Association of atria tissue fibrosis identified by delayed enhancement MRI and atrial fibrillation catheter ablation: the DECAAF study. JAMA 2014ial fibrillation cathet

78. Avicenna. Absence of understanding does not warrant absence of existence. Available at: www.goodreads.com/ quotes/14098-absence-of-understanding-does-not-warrantabsence-of-existence (accessed 11 June 2019).

79. Levinson W, Huynh T. Engaging physicians and patients in conversations about unnecessary tests and procedures: Choosing Wisely Canada. CMAJ. 2014;186:325-6.

80. Thomas $\mathrm{L}$. The dilemma of modern medicine, and the underlying central flaw in medical education and, most of all, in the training of interns, is the irresistible drive to do something, anything. It is expected by patients and too often agreed to by their doctors, in the face of ignorance. Available at: www.brainyquote.com/quotes/lewis_thomas 470012 www.brainycuote.com/qu

81. Grady D, Redberg RF. Less is more: How less health care can result in better health. Arch Intern Med. 2010;170:749-50.

82. Mandrola J, Cifu A, Prasad V, Foy, A. The case for being a medical conservative. Am J Med. 2019. doi: 10.1016/j. amimed 2019.02.005.

83. Fanaroff AC, Califf RM, Windecker S, et al. Levels of evidence supporting American College of Cardiology/American Heart Association and European Society of Cardiology Guidelines, 2008-2018. JAMA. 2019;321:1069-80.

84. Wikiquote. Hooker R. Available at: https://en.wikiquote.org/wiki/ Richard_Hooker (accessed 11 June 2019).

85. Einstein A. A person who never made a mistake never tried anything new. Available at: www.brainyquote.com/quotes/ albert_einstein_148788 (accessed 11 June 2019).

86. Wikiquote. Gide A. Available at: https://en. wikiquote.org/wiki/ Andr\%C3\%A9_Gide (accessed 11 June 2019).

87. Wikiquote. Fatih Sultan Mehmed. Available at: https:// en.wikiquote.org/wiki/Mehmed_ll (accessed 11 June 2019).

88. Galston AW. Nothing that you do in science is guaranteed to result in benefits for mankind. Any discovery, I believe, is morally neutral and it can be turned either to constructive ends or destructive ends. That's not the fault of science. Available or destructive ends. That's not the fault of science. Available at: https://M
June 2019).

89. Husten L. 300+ years later and nothing has changed. Do we control technology, or does technology control us? Cc @ STurkle. 2018. Available at: https://twitter.com/cardiobrief/ status/995697349740679173 (accessed 11 June 2019).

90. De Deyn PP, D'Hooge R. Placebos in clinical practice and research. J Med Ethics. 1996;22:140-6.

91. Wikipedia. Hippocratic Oath. Available at: https://en.wikipedia. org/wiki/Hippocratic_Oath (accessed 11 June 2019).

92. Wikipedia. Suleiman the Magnificent. Available at: https:// en.wikipedia.org/wiki/Suleiman_the_Magnificent (accessed 11 June 2019).

93. Haïssaguerre $\mathrm{M}$, Jaîs $\mathrm{P}$, Shah $\mathrm{DC}$, et al. Spontaneous initiation of atrial fibrillation by ectopic beats originating in the pulmonary veins. N Eng/ J Med. 1998:339:659-66.

94. Chen SA, Hsieh MH, Tai CT, et al. Initiation of atrial fibrillation by ectopic beats originating from the pulmonary veins: electrophysiological characteristics, pharmacological responses, and effects of radiofrequency ablation. Circulation. 1999;100:1879-86

95. Kucuk U. Dr. PACKER: What does or doesn't CABANA say about? \#CABANA @MayoClinicCV. 2018. Available at: https:// twitter.com/ugurkucuk/status/1049287829963067393 (accessed 11 June 2019).

96. Stuart M. CABANA: When clinical trials cause confusion, not clarity. Available at: www.medtechstrategist.com/mts-blog/ cabana-catheter-ablation (accessed 11 June 2019).

97. Velagapudi P. \#Prevention is \#Key!!! \#EmergingLeaders2018. 2018. Available at: https://twitter.com/Pooh_Velagapudi/ status/997022310644137984 (accessed 11 June 2019).

98. Mccabe PJ. What patients want and need to know about atrial fibrillation. J Multidiscip Healthc. 2011;4:413-9.

99. Centor RM. To be a great physician, you must understand the whole story. MedGenMed. 2007;9:59.

100. Jacobs AK, American Heart Association. Rebuilding an enduring trust in medicine: a global mandate: presidential address American Heart Association Scientific Sessions 2004 Circulation. 2005;111:3494-8.

101. Parwani P, Choi AD, Lopez-Mattei J, et al. Understanding socia media: opportunities for cardiovascular medicine. J Am Coll Cardiol. 2019;73:1089-93.

102. Mandrola J, Futyma P. The role of social media in cardiology. Trends Cardiovasc Med. 2019. doi: 10.1016/.ttcm.2019.01.009.

103. Gibson CM. The democratization of medical research and education through social media: the potential and the peril. JAMA Cardiol. 2017:2:9-11. 